\title{
Editorial
}

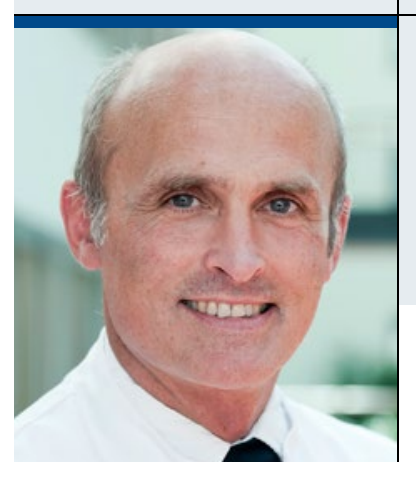

"Bei manchen Patienten können wir zentripetal agieren,

bei anderen müssen wir zentrifugale Kräfte eindämmen."

Prof. Dr. med. Andreas J. Gross

Chefarzt der Klinik für Urologie, Asklepios Klinik Barmbek, Hamburg

\section{Die Blase gehört den Urologen}

$\mathrm{E}$ igentlich möchte man annehmen, dass die Harnblase ein genuin urologisches Organ ist. Patienten mit einer Hämaturie kommen in der Regel auch unverzüglich und ohne Umwege über den Hausarzt in die urologische Praxis. Die ersten diagnostischen Schritte sind einfach und in der Regel zielführend. Die Masse der Patienten, bei denen dann ein Harnblasenkarzinom diagnostiziert wird, hat einen oberflächlichen Tumor. Reseziert - gegebenenfalls nachreseziert - wird er in der urologischen Praxis weiterbetreut.

$\mathrm{Ob}$ wir durch neue diagnostische Maßnahmen früher, mehr oder andere Harnblasenkarzinome entdecken und ob dies den Progress oder die Prognose ändert, werden Untersuchungen zeigen. In dieser Ausgabe der URO-NEWS stellt Christopher Netsch neue Techniken zur Verbesserung der TUR-B und eine dazu gerade anlaufende Studie vor.

Spannend bleibt es beim muskelinvasiven Harnblasenkarzinom, weil der Patient sich entscheiden muss, ob er sich früh oder spät zystektomieren lässt. Neuerdings kommen hier die Onkologen auf den Plan. Sie empfehlen auf dem Boden einer eher dünnen Datenlage eine neoadjuvante Chemotherapie. Auf Rückfrage geben sie zwar zu, dass ihnen wohl bewusst ist, dass eine erhebliche Zahl von Patienten damit übertherapiert wird, aber das Geschäftsmodell eben zu verlockend sei. Interessanterweise weigert sich dieselbe Gruppe jedoch, bei einer Lymphangiosis carcinomatosa eine adjuvante Therapie anzubieten.

Die Nachsorge der zystektomierten Patienten ist aufwendig und sollte der Situation angepasst wer- den. Man ist geneigt, eher zu viel und zu häufig Untersuchungen zu machen. Ann Kathrin Orywal hat sich deswegen mit dem Thema auseinandergesetzt. Sie gibt Kollegen einen Plan an die Hand, zu welcher Zeit welche Maßnahme sinnvoll ist. Es sprechen also viele Argumente dafür, dass Patienten mit Harnblasenkarzinom von Urologen versorgt werden sollten.

\section{Andere Disziplinen vertrauen Urologen}

Patienten mit einer Blasenfunktionsstörung sind auch genuin urologisch. Hier gestaltet sich die Situation jedoch meistens anders. Das Thema ist diffizil und die Behandlungserfolge sind nicht so schnell zu erreichen wie bei den Problemen, die chirurgisch gelöst werden können. Daher werden diese Patienten auch gerne von Kollegen aus anderen Disziplinen zum Urologen weitergeleitet.

Aber auch die Neurourologie folgt handwerklichen Grundregeln und mit dem Armentarium, das uns zur Verfügung steht, können wir Patienten mit angeborener oder erworbener Blasenfunktionsstörung gut helfen.

Bei manchen Patienten können wir also zentripetal agieren, bei anderen müssen wir zentrifugale Kräfte eindämmen.

Fazit: Die Blase bleibt urologisch. Das steht fest.

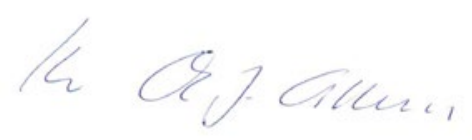

